\title{
'These sorts of people don't do very well': race and allocation of health care resources
}

\author{
Michael Lowe, Ian H Kerridge, and Kenneth R Mitchell fohn Hunter Hospital and University of Newcastle, \\ NSW, Australia
}

\begin{abstract}
Recent literature has highlighted issues of racial discrimination in medicine. In order to explore the sometimes subtle influence of racial determinants in decisions about resource allocation, we present the case of a 53-year-old Australian Aboriginal woman with end-stage renal failure. The epidemiology of renal failure in the Australian Aboriginal population and amongst other indigenous peoples is discussed.

We show that the use of utilitarian outcome criteria for resource allocation may embody subtle racial discrimination where consideration is not given to issues of justice, race, culture and gender. It is only where the processes by which resources are allocated are transparent, clearly defined and based upon consultation with individual patients that issues and justice are likely to be adequately addressed.
\end{abstract}

$\mathrm{CD}$, a 53-year-old Australian Aboriginal woman, was admitted from a regional hospital with pulmonary oedema and worsening renal failure. She had a long history of hypertension, hyperlipidaemia and type- 2 diabetes mellitus and in the past year had developed cataracts, diabetic retinopathy, intermittent claudication and exercise-induced angina pectoris. CD had been known to have renal disease for over ten years and a renal biopsy two years prior to this admission demonstrated mesangioproliferative glomerulonephritis. Renal failure had progressed to the point that she now required either haemodialysis or renal transplant. Her sister had indicated willingness to donate her kidney. After thorough investigation it was decided that she should not be offered a renal transplant. Her doctor summarised her case with the statement: 'These sorts of people don't do very well... .' Kidney disease and other 'western' diseases common in indigenous peoples belong to a group of diseases we label 'diseases of racism'. In this paper we discuss how

\section{Key words}

Discrimination; race and medical treatment; health care and indigenous people. ostensibly neutral utilitarian criteria used to ration the use of resources for such diseases are biased and may embody subtle forms of racism. We argue that the prevention and successful treatment of such diseases will follow the reversal of the racial inequities that are their root cause.

\section{Aboriginal health}

While her doctors would deny that racism played a role in their medical decision-making, it has been well demonstrated that subtle racial biases influence both clinical reasoning and communication with patientso and other physicians $(1,2)$. Recent American studies on heart disease and invasive cardiovascular procedures identify striking racial differences in both disease prevalence and treatment (3-6). It is almost certain that this is also true of Australia.

Estimates of the Aboriginal population of Australia and the Torres Strait Islands vary between 160,000 and 230,000. Sixty per cent of Australian Aboriginals live in urban areas; forty per cent are under 15 years, and only four per cent are aged 60 years or more $(17,18)$. Causes of morbidity and mortality in this statistically skewed population fall into three main clusters. First, Aboriginals have an extremely high perinatal mortality rate compared to the total Australian population and there is a high prevalence of diarrhoeal, parasitic and respiratory disease in infancy (9-11). Second, among teenagers and those in their early twenties and thirties there is a high incidence of psychiatric disorders, unemployment, poverty, homicides, assaults, suicides and motor vehicle accidents (12). This is associated with a high prevalence of alcohol and other substance abuse (13). Overall, the death rates for young adults are as much as ten times that for the rest of the Australian population (14). Third, by early middle age Aboriginals become susceptible to a cluster of 'western' diseases including obesity, Type 2 diabetes mellitus, hypertension and ischaemic heart disease (15). A high proportion of adults smoke and abuse alcohol, and there is a high incidence of liver, lung and cervical cancer $(16,17)$.

Australian Aboriginals share their vulnerability to these diseases with other racial groups that have 
undergone similarly rapid lifestyle and cultural changes such as Native Americans and Pacific Islanders $(10,18)$. A number of interrelated factors can be identified that contribute to this pattern of illness. The main factors identified by Aboriginal groups themselves are: destruction of Aboriginal culture (19); limited education about health and nutrition; high levels of unemployment and lowstatus jobs, and alienation from their land (20). Other factors include inadequate housing, inadequate electricity and sewerage systems, a sedentary lifestyle, high rates of alcohol abuse, geographical isolation, and a heavy dependence upon social welfare (21).

Altogether, Aboriginal death rates are two to four times those of the total Australian population. The calculated life expectancy at birth is 55.2 years for Aboriginal males and 63.6 years for Aboriginal females (22), both figures being 15-20 years lower than the national average and comparable with life expectancies in India, Haiti and Colombia (23).

\section{COMPARISON WITH NATIVE AMERICANS}

Native Americans, while culturally dissimilar to Australian Aboriginals, have much in common. Both groups have been discriminated against, dispossessed of their lands and have suffered informal 'ethnic cleansing'. Both groups have also been subjected to government policies that oscillated between forced assimilation and extermination. While patterns of mortality and morbidity among Australian Aborigines and Native Americans are similar, the incidence and prevalence of disease is consistently higher among Australian Aborigines (24). Recently, both groups have seen a decrease in infant mortality and morbidity due to infections and parasitic diseases but both groups have experienced a marked increase in 'degenerative diseases' such as diabetes, obesity, violence and alcoholism (24).

\section{Renal disease, dialysis and transplantation}

It is not clear why Australian Aboriginals, Pacific islanders and Native Americans share an extreme susceptibility to chronic renal disease (25-27). Contributing factors may include a high prevalence of diabetes, hypertension, vesico-ureteric reflux and glomerulonephritis. Post-streptococcal Glomerulonephritis, Systemic Lupus Erythematosis (28) and IgA Nephropathy have also been reported as significant causes of end-stage renal failure in these populations (29). Aboriginal people are recruited to end-stage renal disease (ESRD) programmes at seven times the rate of other Australians and, on average, ten years earlier (30). It is likely that these figures are in fact an underestimate of the true incidence of ESRD in Aboriginals, due to the remoteness of Aboriginal populations, frequent lack of diagnosis and differing perceptions between
Aboriginals and the health providers of the benefits of treatment (30). Although the causes of ESRD differ between Australian Aboriginal and the total Australian population, once they begin dialysis Aboriginals have a similar prognosis to other Australians. Their life expectancy at one, three and five years ( 89 per cent, 70 per cent, and 55 per cent), is not significantly different from the non-Aboriginal population. Since Aboriginal patients come to dialysis on average ten years before non-Aboriginals however, this represents a markedly shortened lifespan.

In contrast, following renal transplantation, survival rates are significantly lower for Aboriginal than for non-Aboriginal patients. One year survival is approximately 93 per cent for both groups but thereafter the life expectancies of both groups become increasingly divergent. At three and five years, life expectancies are 74 per cent and 60 per cent for Aboriginals compared to 90 per cent and 80 per cent for non-Aboriginals (30). Graft survival shows a similar pattern. Decreased graft survival becomes apparent in Aboriginal patients between one and two years post-transplant and only 20 per cent of grafts survive seven years (30). It is possible that the reason for this difference in survival lies in poor compliance with immunosuppressive medica- 8 tions after the first year of transplant, as similare patterns are seen in non-Aboriginal patients who comply poorly with treatment (31).

\section{NON-COMPLIANCE}

It has been frequently noted that Aboriginal patients comply poorly with medical treatment including hospitalisation, medication and dietary or lifestyle advice. Although inadequately studied, concerns about compliance are sometimes cited when Aboriginals are denied certain types of medications or medical interventions. Dismissal of a patient's claim to a scarce resource such as a kidney on the basis of 'poor compliance', may reflect a superficial understanding of compliance. In many instances issues of compliance are inextricably bound to paternalistic conceptions of clinical decision-making and resource allocation. Non-compliance - the extent to which a patient's behaviour does not coincide with medical or health advice - may occur for a number of reasons, including the inability to understand instructions due to illiteracy or language differences, and inadequate information transfer between the physician and patient. Non-compliance may also reflect more subtle and complex issues such as cultural differences in beliefs about health and disease and unspoken antagonism between doctor and patient (32). Furthermore, for patients such as $C D$, disenfranchised within an alien and hostile environment, non-compliance may represent her only means of expressing her autonomy.

Consideration of these complexities is essential where issues of compliance are identified as being of 
importance to an entire population such as Aboriginals. While for an individual, non-compliance may reflect a number of underlying personal issues, for Aboriginal culture as a whole it may represent a larger division between black and white. Where non-compliance becomes of concern in patient management it should therefore not be taken at face value, rather the underlying assumptions of both doctor and patient need to be thoroughly explored and addressed sensitively in each case.

\section{CRITERIA FOR ALLOCATING KIDNEYS}

Patients in whom renal transplantation or dialysis is clinically indicated are then prioritized for treatment due to the scarcity of resources. It is at this point that individual value judgments most easily enter into the decision-making process, either consciously or unconsciously.

Inequitable access to medical resources may reflect subtle forms of racial discrimination. American studies of patients undergoing renal transplantation and dialysis from 1986 and 1988 showed marked under-representation of non-whites in relation to their representation in the total population of patients with ESRD (33-35). A similar phenomenon has been documented in Britain where members of the upper socioeconomic classes found ways to bypass local limitations on the availability of treatment for ESRD and thus obtain a disproportionate share of medical resources (36).

Utilitarian criteria are commonly used to select patients who may be suitable for renal transplantation. Such criteria seek to identify recipients most likely to benefit (ie, to 'do well') from kidneys where such resources are limited. Outcome criteria that are commonly used to identify benefit include likely graft survival, patient survival and quality-of-style measures. The presence of significant co-morbidity or disability is taken into account. Such utilitarian criteria may embody subtle racial discrimination. As discussed above, Aboriginals as a group have very high levels of cardiovascular disease, diabetes and other illnesses, factors that may prejudice their acceptability for organ transplantation. Similarly utilitarian criteria may discriminate against those who are poor, who are poorly compliant with therapy, who have inadequate access to health services or present late in the course of their disease. Minority-group members and persons of lower socioeconomic status may also lack the education and social skills needed to 'work the system' and surmount barriers to access.

Most importantly, the outcome measures by which we measure 'success' may in themselves be influenced by our cultural biases. 'Western' culture places an extreme value on longevity, at times at the expense of quality of life. In contrast, 'doing well', for a patient like CD might be defined as living among her own people according to her own customs or on her own land. Longevity or graft survival may have little to do with her concept of success. Thrice weekly dialysis conducted at a hospital hundreds of kilometres from family and social support may also have little to offer such a patient despite greater longevity.

\section{Race as a health consideration}

While it is important to be sensitive to issues of culture and race, there are significant dangers in considering such issues when a society has deeply incorporated beliefs about the inherent inferiority of one race and the superior status of another. Such considerations may provide more burdens than benefits for a stigmatised group, even where the stated goal is to help that group (37). As Patricia King has observed: 'If the racial difference is ignored and all groups are treated similarly, unintended harm may result from the failure to recognize racially correlated factors. Conversely, if differences among group or persons are recognized and attempts are made to respond to past injustices or special burdens, the effort is likely to support existing negative stereotypes that contributed to the emphasis on racial differences in the first place.' (38) Race is a complex construct with both biological and social connotations. Significant harm has occurred in the past fromb imputing biological differences between races rather than by assuming or presuming equality (38).

Despite marked differences in the culture, belief systems and genetic make-up of Australian Aboriginals, Pima Indians and other indigenous groups, all have similar health problems. What these groups do have in common is the experience of racism. Perhaps then, these diseases are best thought of as the diseases of racism. Is it then racist to acknowledge the race or culture of one's patient and to some extent base one's treatment on that? Clearly it may be. Equally though, it is also inappropriate to ignore such factors and the difficulties that members of minority groups may face in society. Such issues are extremely subtle and divisive, nevertheless, if they are ignored, then selection criteria that embody inherently racist assumptions may become convenient tools to support policies and behaviour that further disadvantage already vulnerable groups.

\section{What can be done}

Consensus about the definition of reasonable outcome criteria is unlikely to be reached by the varied racial, cultural and religious groups that make up a modern, pluralistic society. If utilitarian outcome criteria are used to allocate health resources without consideration of issues of justice or the process of allocation then they will inevitably embody or reinforce unjust forms of discrimination. Outcome criteria for resource allocation cannot be determined without consideration of issues of power, gender, race and culture. 
Since choices have to be made in order to allocate resources, then the emphasis must lie with the process by which these choices are made rather than with any simplistic statement of desirable outcome criteria. In order for resources to be allocated justly, the process must be centred on shared decisionmaking with individual patients and their carers. The process must be transparent and open to scrutiny and criticism by all members of society, by patients and by health care professionals. Where the aims and goals of medicine are defined openly in consultation between patients and multidisciplinary health care teams, then they are more likely to be just and unbiased.

Thus, in relation to this case, it is vital to establish the key issues and concerns as seen from an Aboriginal perspective. Language difficulties, poor communication, a lack of cultural sensitivity and an inadequate understanding of the health needs and social conditions of Aboriginal people are major barriers to appropriate health care. The perception of many Aboriginal people is that they receive inappropriate treatment in a cultural environment that is not only insensitive to them as people, but also to their culture, values and needs. For many, the experience of being a hospitalized patient is uncomfortable, bewildering and anxiety-provoking. Indeed the provision of health services may be seen by Aboriginals as part of white Australia's colonization policy $(39,40)$.

If public health intervention strategies are to be successful then they must be developed and controlled by Aboriginal communities themselves. In this way services become culturally appropriate, sensitive to the needs and beliefs of Aboriginal people, and 'owned' by the community rather than imposed from the outside (41). It is only when the dispossession that is due to racism is reversed that we as health care professionals can ever hope to have on impact on the diseases of racism. As was noted in the Royal Commission into Aboriginal Deaths in Custody: '.. the elimination of disadvantage requires an end of domination and an empowerment of Aboriginal people; that control of their lives, of their communities must be returned to Aboriginal hands' (42).

\section{Conclusion}

The case of CD illustrates some of the ways that issues of race may influence medical treatment. The burden of disease that affects CD is not only characteristic of Australian Aboriginals but also of indigenous people throughout the world. While these populations differ in many respects, all share the experience of racism and dispossession. Utilitarian criteria for the allocation of health resources may fail to take account of such issues and therefore reinforce racial disparities in health care. We cannot avoid racism by attempting to ignore issues of race.
Communities, including international communities, need to find ways of realistically addressing such issues. In the long term the answers to these problems will lie in the reversal of the dispossession of indigenous people. In the short term, the management of patients like CD must be guided by a determined effort to understand her own values, beliefs and needs.

Michael Lowe, B Med, is a Medical Registrar at the fohn Hunter Hospital, Newcastle, NSW, Australia. Ian H Kerridge, BA, BMed(Hons), MPhil, is Clinical Lecturer, Health Law and Ethics Programme, Faculty of Medicine, University of Newcastle, NSW, Australia. KR Mitchell, MSc, MED, PhD, HGrad Dip Rel St, FAPsS, is Senior Lecturer, Health Law and Ethics Programme, Faculty of Medicine, University of Nerwcastle, NSW, Australia.

\section{References}

(1) Ayanian J Z. Heart disease in black and white. New England journal of medicine 1993; 329: 656-658.

(2) Finucane T E, Carrese J A. Racial bias in presentation of cases. Fournal of general internal medicine 1990; 5: 120-121.

(3) Becker L B, Han B H, Meyer P M et al. Racial differences in the incidence of cardiac arrest and subsequent survival. New England journal of medicine 1993; 329: 600-606.

(4) Whittle J, Conigliaro J, Good C B, Lofgren R P.C Racial differences in the use of invasive cardiovascular? procedures in the Department of Veterans' Affairs medical system. New England journal of medicine 1993; 329: 621-627.

(5) Ayanian J Z, Udvarhelyi I S, Gatsonis C A, Pashos C L, Epstein A M. Racial differences in the use of revascularization procedures after coronary angiography. Fournal of the American Medical Association 1993; 269: 2642-2646.

(6) Gittelsohn A M, Halpern J, Sanchez R L. Income, race, and surgery in Maryland. American journal of public health 1991; 81: 1435-1441.

(7) Thomson N J. Aboriginal health: current status. Australia and New Zealand journal of medicine 1984; 14: 705-718.

(8) Thomson N J. Recent trends in Aboriginal mortality. Medical journal of Australia 1991; 154: 235-239.

(9) Zimmet P, Dowse G, Finch C, Serjentson S, King H. The epidemiology and natural history of NIDDM lessons from the South Pacific. Diabetes metabolic review 1990; 6: 91-124.

(10) O'Dea K. Westernisation, insulin resistance and diabetes in Australian Aborigines. Medical journal of Australia 1991; 155: 258-264.

(11) Peach H. Chronic non-specific respiratory disease in Aborigines. Aboriginal health information bulletin 1988; 10: 24-31.

(12) Radford A J, Harris R D, Brice G et al. Social health among urban Aboriginal heads of households in Adelaide, with particular reference to suicide attempt. Aboriginal health information bulletin 1991; 15: 20-25.

(13) Lake P. Alcohol and cigarette use by urban Aboriginal people. Aboriginal health information bulletin 1989; 11: 20-22. 
(14) Cordner S. The royal commission into Aboriginal deaths in custody: aspects of medical interest. Medical journal of Australia 1991; 155: 812-818.

(15) Hollows F C. Some aspects of Aboriginal health. Australian family physician 1986; 15: 884-887.

(16) Golds M, James R, van Beurden E, Henrikson D. Cardiovascular risk factors in the Aboriginal and islander population of the North Coast, New South Wales. Aboriginal health information bulletin 1991; 16: 20-33.

(17) Hogg R. Aboriginal mortality in western New South Wales during the 1980s. Aboriginal health information bulletin 1991; 16: 38-46.

(18) Knowler W C, Pettit D J, Saad M F, Bennett P H. Diabetes mellitus in the Pima Indians: incidence, risk factors and pathogenesis. Diabetes metabolic review 1990; 6: 1-27.

(19) Trigger D S, Anderson C, Lincoln R A, and Matis C E. Mortality rates in 14 Queensland Aboriginal reserve communities: association with 10 socioeconomic variables. Medical journal of Australia 1983; 1: 361-365.

(20) Gracey M, Sullivan H, Spargo R. Economic factors affecting Aboriginal health and health care. Aboriginal health information bulletin 1988; 9: 36-49.

(21) Australian Bureau of Statistics. Census of Population and Housing, Small area data. Canberra, Australia, 1986.

(22) Australian Government Publishing Service. Australia's health 1992: the third biennial report of the Australian Institute of Health and Welfare. Canberra, 1992: 210-218.

(23) United Nations Children's Fund. The state of the world's children, 1988. Oxford: Oxford University Press, 1988.

(24) Kenen R H. Health status: Australian Aborigines and Native Americans - a comparison. Australian Aboriginal studies $1987 ; 1: 34-45$.

(25) Van Buynder P G, Gaggin J A, Mathews J D. Renal disease patterns in Aboriginal Australians. Medical journal of Australia 1993; 159: 82-87.

(26) May W E, Megill D M. End-stage renal disease in southwestern Native Americans, with special focus on the Zuni and Navajo Indians. Transplant proceedings 1989; 21: 3906-3908.

(27) Neale T J, Bailey R R. Chronic renal disease in Polynesians in New Zealand. New Zealand medical journal 1990; 103: 262.

(28) Anstey N M, Bastian I, Dunckley M, Currie B J.
Systemic lupus erythematosus in Australian Aborigines: high prevalence, morbidity and mortality. Australia and New Zealand journal of medicine 1993, 23: 646-651.

(29) O'Connell P J, Isbels L S, Thomas M A, Harris M, Eckstein R P. Familial IgA Nephropathy: a study of renal disease in an Australian Aboriginal family. Australia and New Zealand journal of medicine 1987; 17: 27-33.

(30) Disney A P S, ed. Fifteenth report of the Australian and New Zealand Dialysis and Transplant Registry (ANZDATA). 1992, Oct.

(31) Arnason W B. Directed donation: the relevance of race. Hastings Center report 1991; 21: 13-19.

(32) Holm S. What is wrong with compliance? fournal of medical ethics 1993; 19: 108-110.

(33) Levinsky N G. Lessons from the Medicare End-stage Renal Disease Program. New England journal of medicine 1993; 329, 9: 1395-1399.

(34) Gaylin D S, Held P J, Port F K, et al. The impact of comorbid and sociodemographic factors on access to renal transplantation. Fournal of the American Medical Association 1993; 269: 603-608.

(35) Kasiske B L, Neylan J F III, Riggio R R, et al. The effect of race on access and outcomes in transplantation. New England journal of medicine 1991; 324: 302-307.

(36) Kjellstrand C M. Age, sex, and race inequality in renal transplantation. Archives of internal medicine 1988; 148: 1305-1309.

(37) Caplan A L. When evil intrudes. Hastings Center repor 1992; 22: 29-32.

(38) King P A. The dangers of difference. Hastings Centes report 1992; 22: 35-38.

(39) Bartlett B. Central Australia: medical practice in Australia's fourth world. Medical journal of Australia 1991; 155: 470-472.

(40) Whimp K, McDonald D. The national report of the royal commission into Aboriginal deaths in custody (RCIADIC): findings relating to Aboriginal and Torres Strait Islander health. Aboriginal health information bulletin 1991; 16: 14-19.

(41) Foley G. Aboriginal community controlled health services - a short history. Aboriginal health information bulletin 1982; 2: 13-15.

(42) Johnston E, Commissioner. Royal commission into Aboriginal deaths in custody: national report [5 vols]. Canberra, AGPS: 1991: para 1.7.6. 